\title{
Indolent pneumonia in a pregnant recent immigrant from Ethiopia: think TB
}

\author{
*Hashem Bishara1, Moshe Lidji², Olga Vinitsky³, Daniel Weiler-Ravell1 \\ ${ }^{1}$ Tuberculosis Center, Nazareth Hospital, Nazareth, and Faculty of Medicine in Galilee Bar-Ilan University, Israel \\ 2 Tel Aviv Tuberculosis Clinic, Israel Lung Association, Tel Aviv, Israel \\ ${ }^{3}$ Ministry of Health Northern District, Israel
}

Originally received 19th August 2013; resubmitted 6th October 2013; revised 3rd November 2013; accepted 10th November 2013; online 27th January 2014

\begin{abstract}
Summary made, and the dilemma of initiating treatment in unconfirmed TB.

(C) 2014 Primary Care Respiratory Society UK. All rights reserved.

H Bishara et al. Prim Care Respir J 2014; 23(1): 102-105

http://dx.doi.org/10.4104/pcrj.2014.00001
\end{abstract}

Time delay to tuberculosis (TB) diagnosis remains a public health concern. In pregnancy, early TB diagnosis is challenging and acquires further significance due to the risk of infection of the newborn as well as others in the maternity setting. We report a delay of 12 weeks in the diagnosis of TB in a pregnant recent immigrant from Ethiopia to Israel. Contact investigation revealed pulmonary TB in her two daughters aged four and seven years. We discuss the reasons for this delay in diagnosis, how a more timely diagnosis might have been

Keywords case history, diagnosis, pneumonia, pregnancy, tuberculosis

\section{Introduction}

Tuberculosis (TB) is a leading cause of mortality worldwide, mainly in developing countries. It is also an ongoing problem in developed countries due to migrant populations from regions with high TB prevalence and other high TB risk groups. Early recognition and treatment of active pulmonary TB disease are the most important factors in containing TB and preventing its spread. Diagnosis of TB in pregnancy is challenging because the non-specific symptoms of early TB such as weakness, excessive perspiration, and tachycardia will usually be attributed to the pregnancy. Primary care physicians are usually the first to encounter these patients, and therefore can contribute to early diagnosis.

\section{Case presentation}

A 28-year-old recent immigrant from Ethiopia to Israel in her 26th week of pregnancy was examined for a persistent cough of three weeks duration with no fever and scant sputum production. She was in a good general condition, without tachypnoea, and had normal breath sounds. Her symptoms persisted and two weeks later a chest $x$-ray (CXR) showed a paracardial infiltrate in the left midlung field (Figure 1). Since she did not improve with antibiotic treatment, the possibility of TB was considered at her follow-up examination four weeks later. Since the regional TB centre was

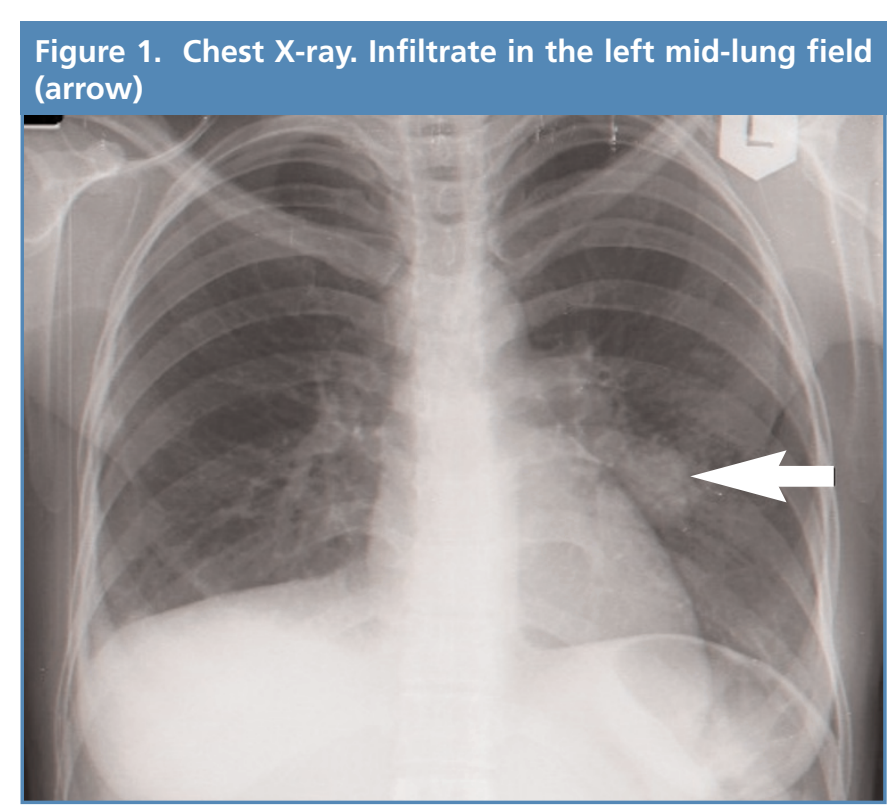

$60 \mathrm{~km}$ away, she was referred to the local hospital and was admitted to an obstetric ward. On admission she was afebrile, not in acute distress, with a regular pulse of 104 and blood pressure of 110/66. There was no change in the finding in the CXR and her sputum was

\footnotetext{
* Corresponding author: Dr Hashem Bishara, Tuberculosis Center, Nazareth Hospital, POB 422, Nazareth 16100, Israel.

Tel: +97246028888 Fax: +97246028857 E-mail: hbishara@bezeqint.net
} 
smear-negative for acid-fast bacilli. She was treated again with broad spectrum antibiotics and discharged after a week. Two weeks later she coughed up blood and was referred to the TB clinic. Considering her ethnic background, the persistent cough, haemoptysis, and the non-resolving pulmonary infiltrate, a clinical diagnosis of TB was made and treatment with isoniazid (INH), rifampicin (RIF), and ethambutol (EM) was begun. Later, her sputum culture returned positive for TB, sensitive to all first-line drugs.

At 36 weeks she delivered at home, so the placenta could not be examined for the presence of granulomas or acid-fast bacilli. The $2115 \mathrm{~g}$ infant was examined on the following day in the newborn department. On medical examination he had no fever, respiratory distress, lymphadenopathy, or hepatosplenomegaly. His CXR and routine blood tests including liver enzymes were normal. The infant was breast-fed with his mother using a mask. Directly observed treatment with INH for possible latent TB infection was started but six months later the treatment was stopped following a negative tuberculin skin test (TST).

\section{Investigation and management of contacts}

The patient had three close household contacts who were examined and who had investigations performed including a TST and a CXR.

Her asymptomatic 7-year-old daughter had a positive 22mm TST and her CXR revealed a loculated right pleural effusion (Figure 2). She was admitted to the paediatric ward for further investigation. A diagnostic thoracentesis was performed but only a small amount of exudative pleural fluid was drained, with a leucocyte count of $1800 / \mu l$, predominantly (60\%) lymphocytes. Because of the small amount of pleural fluid drained, TB culture and measurement of adenosine deaminase levels could not be performed. Sputum and gastric aspirate were collected for smear and TB culture. Chest computed tomography showed a loculated right pleural effusion (Figure 3) and mediastinal lymphadenopathy with no pulmonary parenchymal infiltrate (Figure 4). Treatment for TB was initiated with a four first-line drug regimen (INH, RIF, pyrazinamide (PZA), and EMB). The effusion eventually resolved without any sequelae. Cultures of her sputum and gastric aspirate were never positive.

The younger 4-year-old daughter who coughed persistently had a $25 \mathrm{~mm}$ positive TST and left upper and lower lobe infiltrates on her CXR. Treatment for TB was started with the four first-line drugs and the diagnosis was confirmed later by a smear-negative TB-positive sputum culture sensitive to all first-line drugs.

Her TST-positive husband was treated for latent TB with twice weekly directly observed preventive therapy (DOPT) of 900mg INH for nine months (a standard Centers for Disease Control (CDC) regimen which facilitates DOPT). Treatment was stopped prematurely after 46 doses (total required 76) because of INHinduced hepatitis. The family moved shortly afterwards and treatment of the latent TB was never resumed. Two years later he had increasing abdominal pain of several weeks duration, was examined and discharged twice from an emergency department until, finally, on his third referral after high fever and ascites had developed a diagnosis of abdominal TB was made on laparoscopy.
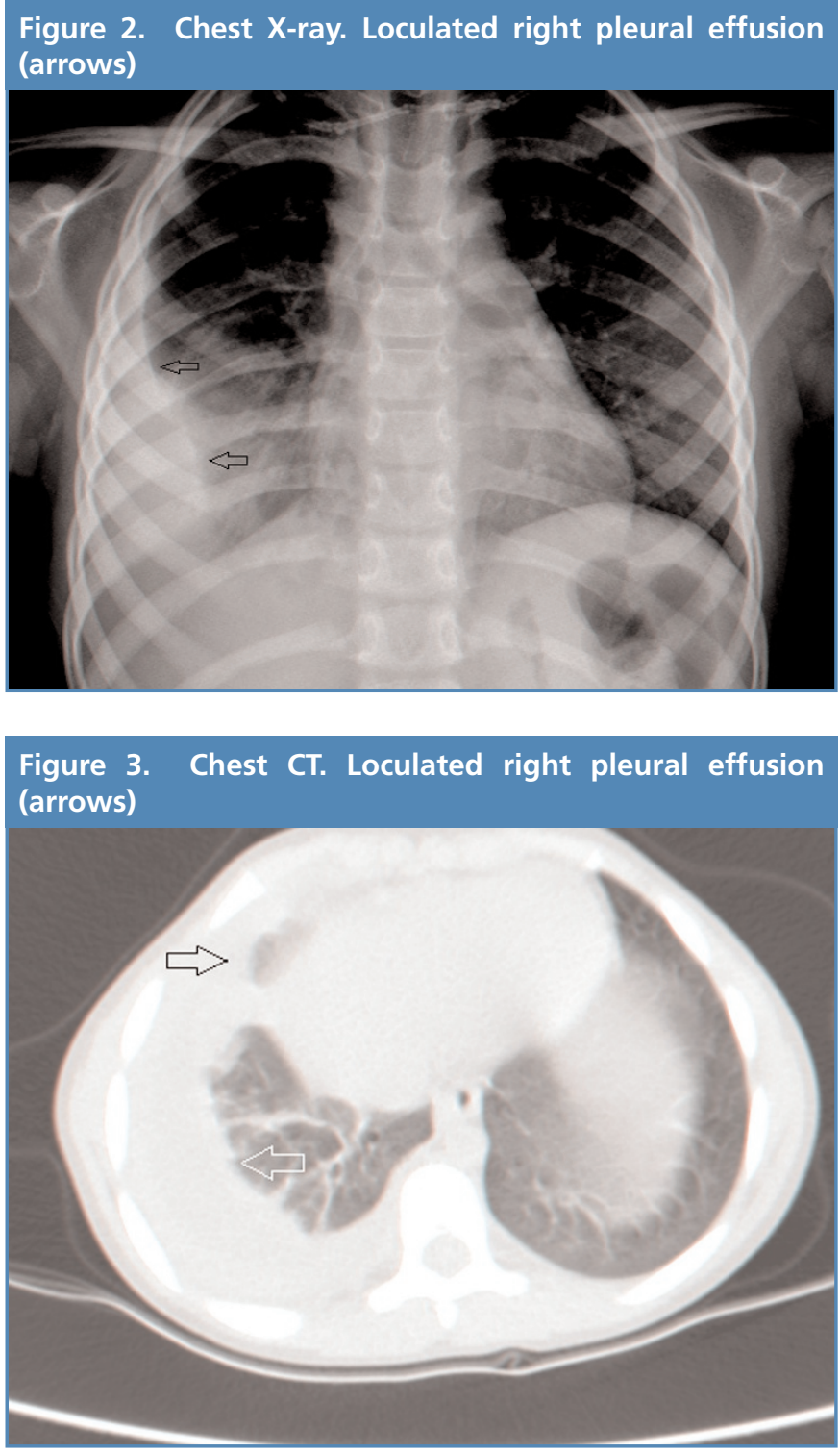

Figure 4. Chest CT. Right paratracheal lymphadenopathy (arrow)

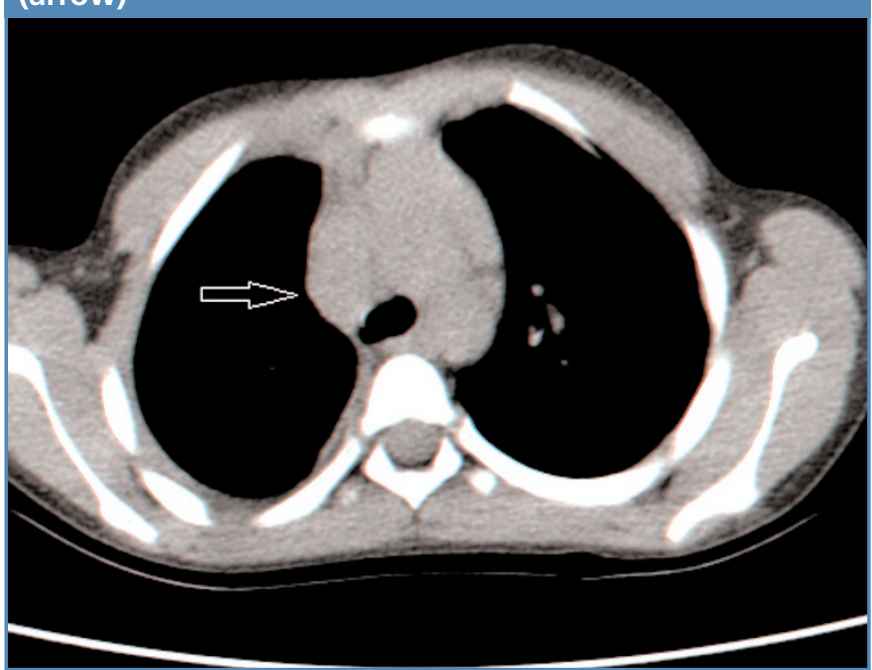


Peritoneal fluid and biopsy cultures were positive for Mycobacterium tuberculosis sensitive to all first-line drugs. The patient was treated with a first-line four-drug regimen with directly observed treatment at the regional TB centre and eventually cured.

DNA finger printing using a 24 loci-based typing scheme (MIRUVNTR) showed a genetically identical $M$. tuberculosis strain for all three culture-positive cases. All patients were HIV-negative and all fully recovered with standard therapy.

\section{Discussion}

The likelihood that indolent pneumonia in recent immigrants (up to five years after arrival) from countries with a high TB burden may be TB should always be considered. ${ }^{1}$ A negative sputum smear does not rule out the diagnosis of active TB if TB is suspected. In such cases, starting treatment while awaiting culture results is often warranted, especially in children and immunocompromised individuals such as those who are HIV-positive. However, presumptive treatment of bacteriologically unconfirmed TB demands additional diagnostic efforts.

Community-acquired pneumonia is far more common than TB in low burden countries. ${ }^{2}$ However, as this case history illustrates, physicians will miss the diagnosis of TB if they do not take ethnic origin into consideration. This is particularly true in countries where TB is rare but with pockets of high-risk groups where rates are much higher. ${ }^{3}$ Being a recent immigrant from a high burden TB country is a prime risk factor for $T B$, and often subacute non-specific symptoms of respiratory infection imply TB in such patients. The majority of TB patients in Western countries are foreign-born recent immigrants. About $87 \%$ of TB patients in Israel and $74 \%$ of all new TB cases in the UK are foreign-born. ${ }^{4}$ Of 33 cases of TB in pregnant women reported from the UK, all but one were foreign-born and all came from immigrant families. ${ }^{5}$

In the index case presented there was a 12-week delay between the onset of symptoms and diagnosis. This delay was due to:

1) A lack of awareness at the primary care level of the risk of TB in recent immigrants from high burden countries which resulted in a delay in referral.

2) A lack of appreciation of the risk of TB in smear-negative patients when the diagnosis was considered; in the UK, $50 \%$ of active culture-positive TB is smear-negative. ${ }^{4}$

3) The non-upper lobe location of the infiltrate was misleading; atypical lower lobe infiltrates, hilar lymphadenopathy, and pleural effusion may be found in primary pulmonary TB, particularly in HIV-positive patients where a miliary pattern is also more frequent. ${ }^{6}$

4) Physicians are unjustifiably reluctant to $X$-ray a pregnant patient. Likewise, there is unjustified reluctance to start treatment of TB - particularly in pregnancy - without compelling evidence of active disease. However, if the clinical suspicion is high, a trial of treatment may be justified in the absence of definite proof of disease. The benefits of early treatment far outweigh the risks for the patient, the fetus, and all close contacts. ${ }^{\text {? }}$

5) Barriers to medical care for immigrants. These include unique health beliefs and practices, fear of stigmatisation, difficulty with language and transportation, unfamiliarity with health systems, and poverty (to name a few). ${ }^{8}$ In this case they prevented the patient from reaching the TB clinic. When TB was eventually suspected and the patient referred for evaluation, she did not have the means to reach the TB clinic $60 \mathrm{~km}$ away and ended up instead in a maternity ward at the nearest hospital where, once again, the risk of TB was not appreciated.

According to the International Union against Tuberculosis and Lung Disease and the World Health Organization, patients in countries with a high TB incidence who have been coughing for three weeks or more should be suspected as having TB. ${ }^{9}$ This is an epidemiological recommendation not considered relevant in low burden Western societies. We could learn from this case that this tenet might also be valid for recent immigrants from high TB prevalence countries. Had it been applied here, it certainly would have shortened the time to diagnosis.

In the case of a pregnant patient with TB, the World Health Organization and the British Thoracic Society recommend treating TB with all four first-line drugs (INH, RIF, EMB, and PZA). ${ }^{10,11}$ However, considering the low likelihood (3.5\%; personal communication, $\mathrm{Dr}$ Z Mor, September 2013) of INH drug resistance among Ethiopian immigrants to Israel, ${ }^{12}$ we preferred to abstain from the use of PZA during pregnancy due to lack of adequate information regarding adverse effects on the fetus, in accordance with the CDC recommendations. ${ }^{13}$

Three members of the patient's household contracted active TB despite her being smear-negative. The sensitivity of smear microscopy is highly variable, performing worst in HIV-infected patients and children. ${ }^{1}$ The yield of sputum smears increases with the number of specimens and their adequacy. The current recommendations are to submit three sputum specimens from each patient in whom the diagnosis of TB is being considered but has not yet been established, and to perform at least one nucleic acid amplification test. However, physicians should use clinical judgement whether to begin anti-TB treatment if the acid-fast bacillus smear result is negative. ${ }^{14}$ Obtaining adequate samples is challenging and may be an obstacle to diagnosis, especially in children. Patients should be taught how to expectorate and perform a deep cough. However, transmission of TB from smear-negative patients with TB does occur. The likelihood of transmission is greater when the source case coughs persistently, with proximity to the index case, and when living conditions are cramped. ${ }^{15}$ Our case occurred in just those conditions. Once infected, children are at a higher risk of developing active and severe forms of the disease.

While every effort should be made to attain bacteriological confirmation of disease including invasive procedures, in vulnerable populations treatment initiation should not be delayed by waiting for a definite diagnosis. ${ }^{16}$ In the case of the 7-year old-daughter, further investigations such as bronchoscopy or pleural biopsy were considered. However, on the basis of the epidemiological background, the low yield from bronchoscopy when no parenchymal lung involvement was evident on the chest computed tomographic scan, and the possible complications of pleural biopsy in a 7 -year-old $20 \mathrm{~kg}$ child, we proceeded with full anti-TB treatment while awaiting culture results. ${ }^{17}$ 


\section{Learning points}

1. Facing what seems like an indolent atypical pneumonia in recent immigrants from countries with a high TB burden, the possibility of TB should always be considered.

2. When clinically suspected, a negative sputum smear does not rule out the diagnosis of active TB.

3. In primary pulmonary TB and when HIV-positive, pulmonary TB frequently presents with 'atypical' lower lobe infiltrates and hilar lymphadenopathy on CXR.

4. When the clinical suspicion for TB is high, physicians should not hesitate to X-ray a pregnant woman and, in vulnerable populations, start treatment for active TB. The benefits of early treatment in such circumstances far outweigh the risks.

Because it is more difficult to isolate $M$. tuberculosis from a child with pulmonary (particularly pleural) TB than from an adult, the choice of drug treatment for the child is frequently based on the results of culture and susceptibility tests of specimens from the index case presumed to be the source of the infection in the child. ${ }^{17}$ In children in whom drug resistance is suspected or for whom no source case isolate is available, attempts to isolate organisms via three early morning gastric aspirations, bronchoalveolar lavage, or tissue biopsy must be considered. Serological and nucleic acid amplification (e.g. polymerase chain reaction) tests are not currently recommended for routine diagnosis of childhood TB, as they have been inadequately studied in children and have performed poorly in the few studies which have been done. ${ }^{18}$

Young children with uncomplicated pulmonary TB or tuberculous peripheral lymphadenitis who have a low risk of INH resistance and who are HIV-negative can be treated with three drugs (INH, RIF, PZA) during the intensive two-month phase of treatment. ${ }^{19}$ The three-drug regimen is effective in children because of the low bacillary load. However, in children who have extensive or cavitary lung disease or a risk of drug resistance, treatment should consist of a four-drug regimen including EMB during the intensive phase, followed by INH and RIF during the four-month continuation phase.

In passing, it is worth noting the delay in diagnosis in the husband of the index case who presented with abdominal TB two years later. Once again the risk of TB in an immigrant from a high burden country was not recognised. This inherent risk was further compounded by his history of having been a close contact of an active case of TB with a positive TST, who failed to complete treatment of his latent TB. His case also highlights the price that may be paid for not completing treatment of latent TB - a common occurrence, the significance of which is not always fully appreciated by attending physicians. ${ }^{20}$

Our response to the treatment delay in this case has been to provide free and direct transportation to the regional TB clinic for hard-to-reach TB suspects and their high-risk contacts.

\section{Handling editor Jaime Correia de Sousa}

Acknowledgements The authors would like to thank E Rorman, D Goldblatt, and H Kaidar-Shwartz and the National Mycobacterium Reference Laboratory-National Public Health Laboratory, Ministry of Health, Tel-Aviv, Israel for performing the DNA genotyping and their professional advice. The authors also thank $\operatorname{Dr} Z$ Mor for providing the drug resistance data and the patient and her spouse for their approval to publish this case.

Conflicts of interest The authors declare that they have no conflicts of interest in relation to this article.

Contributorship All authors were involved in the conception, drafting, and approval of the final version of the article.

Funding There was no funding for this article.

Patient consent A signed consent form was submitted as part of the original submission on 19th August 2013

\section{References}

1. Davies PD, Pai M. The diagnosis and misdiagnosis of tuberculosis. Int J Tuberc Lung Dis 2008;12:1226-34

2. Welte T, Torres A, Nathwani D. Clinical and economic burden of community-acquired pneumonia among adults in Europe. Thorax 2012;67:71-9. http://dx.doi.org/10.1136/thx.2009.129502

3. Abubakar I, Lipman M, Anderson C, Davies P, Zumla A. Tuberculosis in the UK: time to regain control. BMJ 2011;343:d4281. http://dx.doi.org/10.1136/bmj.d4281

4. Health Protection Agency. Tuberculosis in the UK: annual report on tuberculosis surveillance in the UK, 2012. London: Health Protection Agency, 2012. Available at: http://www.hpa.org.uk/webc/HPAwebFile/HPAweb_C/1317134913404 (accessed 22 May 2013)

5. Knight M, Kurinczuk JJ, Nelson-Piercy C, Spark P, Brocklehurst P. Tuberculosis in pregnancy in the UK. Br J Obstet Gynaecol 2009;116:584-8. http://dx.doi.org/10.1111/j.1471-0528.2008.02097.x

6. Zumla A, Raviglione M, Hafner R, von Reyn CF. Tuberculosis. N Engl J Med 2013; 368:745-55. http://dx.doi.org/10.1056/NEJMra1200894

7. Getahun H, Sculier D, Sismanidis C, Grzemska M, Raviglione M. Prevention, diagnosis, and treatment of tuberculosis in children and mothers: evidence for action for maternal, neonatal, and child health services. J Infect Dis 2012;205:S216-27. http://dx.doi.org/10.1093/infdis/jis009

8. Chemtob D, Weiser S, Yitzhak I, Weiler-Ravell D. Medical anthropology: an important adjunct to international TB control. In: Reichman LB, Hershfeld E, eds. Tuberculosis, a comprehensive international approach, 2nd ed. New York: Marcel Dekker Inc, 2006. p.745-70

9. World Health Organization, International Union Against Tuberculosis and Lung Disease, Royal Netherlands Tuberculosis Association. Revised international definitions in tuberculosis control. Int J Tuberc Lung Dis 2001;5:213-15.

10. World Health Organization. Treatment of tuberculosis: guidelines for national programmes. WHO/HTM/TB/2009.420/

11. Joint Tuberculosis Committee of the British Thoracic Society. BTS guidelines: chemotherapy and management of tuberculosis in the United Kingdom: recommendations 1998. Thorax 1998;53:536-48 http://dx.doi.org/10.1136/thx.53.7.536

12. World Health Organization. Tuberculosis country profiles - Ethiopia. Available at: https://extranet.who.int/sree/Reports?op=Replet\&name=\%2FWHO_HQ_Reports\%2 FG2\%2FPROD\%2FEXT\%2FTBCountryProfile\&ISO2=ET\&LAN=EN\&outtype=html

13. American Thoracic Society, Centers for Disease Control and Prevention and the Infectious Diseases Society. Treatment of tuberculosis. Am J Respir Crit Care Med 2003;167:603-62. http://dx.doi.org/10.1164/rccm.167.4.603

14. Centers for Disease Control and Prevention (CDC). Updated guidelines for the use of nucleic acid amplification tests in the diagnosis of tuberculosis. MMWR Morbidity Mortality Wkly Rep 2009;58(1):7-10.

15. Bloch $A B$. Screening for tuberculosis and tuberculosis infection in high-risk populations: recommendations of the Advisory Council for the Elimination of Tuberculosis. MMWR Recomm Rep 1995;44(RR-11):18-34

16. Perez-Velez CM, Marais BJ. Tuberculosis in children. N Engl J Med 2012;367:34861. http://dx.doi.org/10.1056/NEJMra1008049

17. Starke JR. Tuberculosis in infants and children. In: Tuberculosis and nontuberculous mycobacterial infections. 6th ed. Washington DC: ASM Press, 2011. p.456-75.

18. World Health Organization. Guidance for national tuberculosis programmes on the management of tuberculosis in children. WHO/HTM/TB/2006.371.

19. World Health Organization. Rapid advice: treatment of tuberculosis in children WHO/HTM/TB/2010.13

20. Horsburgh CR Jr, Goldberg S, Bethel J, et al. Latent TB infection treatment acceptance and completion in the United States and Canada. Chest 2010;137:40109. http://dx.doi.org/10.1378/chest.09-0394 